\title{
Development of Virtual Experiment on Operational Amplifier Applications
}

\author{
Bhaskar Y. Kathane, \\ PCD ICSR, VMV College Campus, \\ Wardhaman Nagar, Nagpur (MS), \\ india
}

\author{
Pradeep B. Dahikar \\ Kamla Nehru Mahavidyalaya \\ Sakkardara, \\ Nagpur (MS), India
}

\author{
D. A. Deshpande, \\ PCD ICSR, VMV College Campus, \\ Wardhaman Nagar, Nagpur (MS), \\ India
}

\begin{abstract}
The scope of this paper includes development and implementation of virtual lab for Operational Amplifier. The study of Operational Amplifier is important in Communication Electronics. The Operational Amplifier experiment can be performed by using the concept of virtual Intelligent SoftLab (VIS). The virtual experiment described here will help students to perform it any time anywhere. The screen shows virtual analog or digital waves and shows related outputs with the necessary circuit for studying Operational Amplifier. There is a facility for change of amplitude and frequency of analog and digital signal. The effect of analog and digital signal is also visible on the screen.
\end{abstract}

Keywords: - SoftLab, Operational Amplifier, Virtual, VIS Model

\section{INTRODUCTION}

Science subjects always have a component of practical. In subjects like Physics and Electronics students have to perform a large number of experiments in an academic year. Many times students do not get time to repeat experiments which they have performed during the session. Also many of the laboratories lack in resources to perform experiments in which sophisticated instruments are required. Therefore to provide access to laboratory experiments, anytime anywhere, concept of virtual laboratory is developed. This virtual laboratory can cater to students at under graduate (UG) and post graduate (PG) levels. Some software's like Mat Lab and Lab VIEW are available for simulation of experiments and for other purposes. However, these software's are generally available only in big Institutes/Laboratories and student can use them only during college hours. It is therefore decided to develop software for performing individual experiments virtually on computer screen. An attempt is made to develop software for electronics experiments from basic to advance level. Facilities of the laboratories in educational institutions are generally insufficient when the number of students is considered. Implementation of a laboratory to meet the requirements has a very high price. Because of using intensively software and minimum hardware, virtual laboratories that can be controlled remotely as a real time have become alternative to the traditional laboratories. In these laboratories, students can access to the experiments without time and cost limitations. In the virtual laboratory, students can set up parameters of experiments from a remote location and repeat the experiments, as they need [1].

Question of the performing experiments and lack of resources is being solved to some extent using web based and video based courses [2]. Conducting joint experiments by two participating institutions and also sharing costly resources is also a way out in research. But this is always a challenging task. With the present day internet and computer technologies, the above limitations can no more hamper students and researchers in enhancing their skills and knowledge. Web enabled experiments have been designed for remote operation and viewing so as to enthuse curiosity and innovation into students [3]. This would help in learning basic and advanced concepts through remote e-experimentation. Today most equipment have computer interface for control and data storage. It is possible to design good experiments around some of these equipments which would enhance understanding of a student. Internet-based experimentation further permits use of resources - knowledge, software, and data available on the web, apart from encouraging skillful experiments being simultaneously performed at points separated in space.

In the present work a virtual intelligent SoftLab by simulation technique is developed. The simulation is implemented using VB/VB.Net computer programming language. This simulation gives live feel of experiment through virtual Intelligent SoftLab (VIS). The role of virtual laboratories in education is to help students to improve their quality and capability. The Virtual Laboratory is a platform where user can perform experiment designed to suit a particular course. It is easy to understand why VIS is better as compared to LabVIEW or MatLab for a particular course. VIS provides facility to performed experiments for a particular course any were any time. The VIS collects and presents texts and images concerning various aspects of the experiments. One can develop a whole lot of experiments for a particular course such as experiments with characteristics of devices, Digital Electronics etc. Through VIS students can fully visualize, practice and understand the concept behind the experiment. Working of any Instrument, howsoever complicated, can be visualized and understood. The research work carried out here is intended for better learning and understanding of electronics at college level. The research work is undertaken with following hypothesizes:

- That basic electronics syllabus for practical can be implemented using VIS framework and will be extremely useful to student in understanding several concepts.

- That the VIS frame work shall reduce time spent in the laboratory.

- That the VIS frame work shall decrease students' requests for assistance and,

- That the VIS frame work shall provide high student satisfaction with the laboratory experience.

\subsection{Types of Virtual Labs}

- The Internet Labs: This technology allows students to participate in an experiment remotely as long as they 
have access to high speed Internet. These labs do not need a human interface in the lab to be conducted.

- The Laboratory Website: A lab website is developed using Google Sites for local as well as remote students to demonstrate the experiments.

- Virtual Labs: Engineering students use the virtual lab which is designed by IIT's. Several virtual laboratories developed by other are also available on the web. The National Aeronautics and Space Administration (NASA) Learning Technologies Project (LTP) offers free virtual operation of on-line devices to examine real specimens. These virtual devices include a Scanning Electron Microscope (SEM), Light Microscope, Atomic Force Microscope, and an Energy Dispersive Spectrometer for the SEM. The National Science Digital Library (NSDA) also provides college and high school students around the world with a virtual lab simulator to explore and reinforce fundamental concepts of introductory chemistry.

- LabVIEW: The Laboratory Virtual Instrumentation Engineering Workbench is a system design platform and development environment using visual programming language.

- Circuit Maker: Circuit maker is a downloading tool which is normal user to design and simulate his various circuits using circuit maker.

- MatLab: The Matrix laboratory is a numerical computing environment and fourth generation programming language. MATLAB allows matrix manipulations, plotting of function and data, implementation of algorithms etc

\section{RESEARCH OBJECTIVES}

The important objective of the research is to provide a computer base solution to perform experiments in a Basic Electronics any where any time. This is achieved through developing a VIS platform. The Lab Platform is software developed in this research which needs to be installed for its use. Using this platform the user can run simulations and view results. Another objective is to develop VIS platform for not only basic electronics lab but for digital electronics and communication electronics labs too. This software tool will allow the students to make a circuit, check its correctness or otherwise, perform the experiment and analyze results. It supports the full range of activities required to conduct a complete laboratory course in Basic Electronics. The simulation of each experiment in Basic Electronics is achieved through use of VB/ VB.Net in the VIS platform

\section{VIS Model}

The SoftLab philosophy challenges us to link the physical laboratory experiment with its theoretical simulation model within a unified and interactive environment. The goal for instance of a SoftLab is to create a software environment where experiments are simulated, results is displayed and analyzed.

The VIS model supports both the real class room and the virtual classroom for demonstration purposes. Virtual Laboratory gives virtual instrument to user so that he arranges experimental circuits, set parameters and observes simulated results using Virtual Intelligent SoftLab. The VIS model supports the virtual classroom with experimental methodology so that the user learns about the experiment and performs experiments using virtual instruments [4]. The model used in developing Virtual Intelligent Soft lab is depicted in the figure 2.1

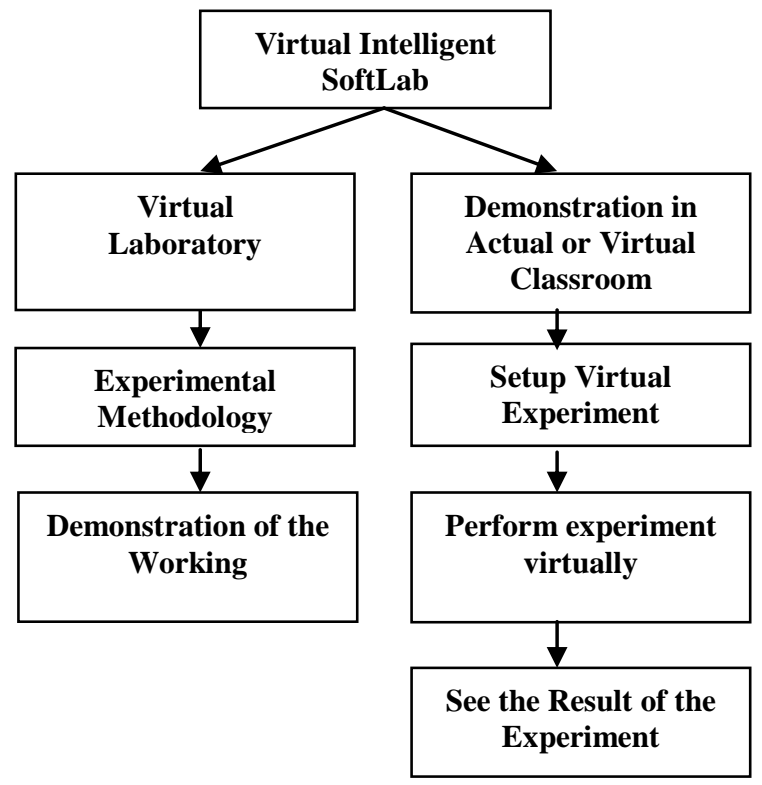

Fig 1: VIS Model

\subsection{System Architecture}

The Virtual Intelligent SoftLab (VIS) model use three-tier system architecture: User, VIS Management System and Database. User Tier is mainly for the user. In this Tier the Students, researcher and Teacher can access the virtual experimental facility from the VIS Management System. VIS Management System Tier is complete software developed in VB/VB.Net for performing electronics experiments. Even instruments such as signal generator, Oscilloscope, Power Supply and components like potentiometer are brought visually on the screen of the computer. Data Tier mainly is the Database. In this tier we have stored data necessary for performing experiments, their result are analysis. The three tier system work as a simulator for experiments.

A facility is also provided in the VIS Management System software whereby an administrator or teacher in the lab first logs in and then other system can be used by students.

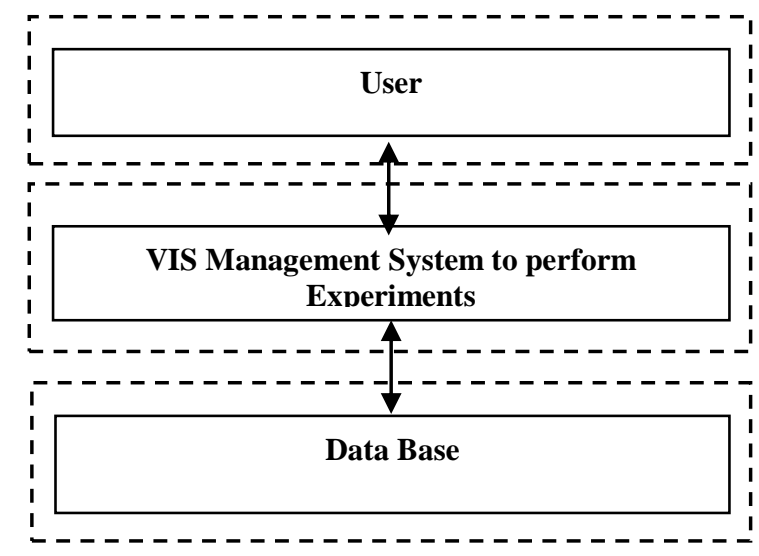

Fig 2: 3-Tier Architecture of VIS 


\subsection{Working flow of VIS}

VIS Management systems basically design for the students or researcher. There are four courses available for user such as Characteristic of Solid state devices, Application of solid state devices, Digital Electronics and Communication Electronics. All courses have the Virtual Classroom (experimental methodology) and the Virtual Laboratory. In virtual classroom we explain the purpose of experiment, experimental procedure and connection help with virtual instruments, possible observation and its application. In Virtual Laboratory we provide virtual Instruments for circuit connection and observed the results on the screen.

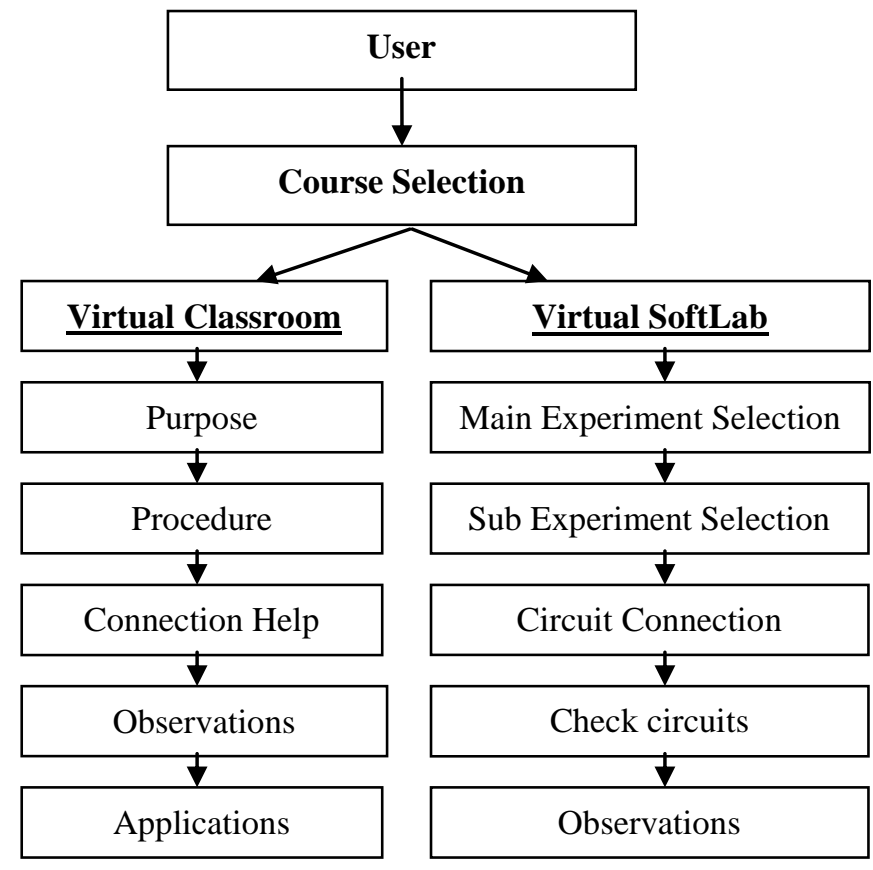

Fig 3: The Main working flow of VIS

\section{METHODOLOGY}

Visual Basic is a third generation event-driven programming language and integrated development environment from Microsoft for its COM programming model. VB is also considered as relatively easy to learn and use programming language, because of its graphical features. Visual Basic was derived from BASIC and enables use of graphics user interface, access to database and creation of ActiveX controls and objects. A programmer can put together the component provided with Visual Basic itself to develop an application. The language not only allows programmers to create simple GUI applications, but can also develop complex applications. Programming in VB is a combination of visually arranging component or control on a form, specifying attributes and actions of those components. Visual Basic can create executables (EXE files), ActiveX control or DLL files, but is primarily used to develop Windows applications. The beauty of this language is that it does not require the Database to manage data.

\section{VIS Implementation for Experiments in Operational Amplifier}

Operational amplifiers are important building blocks for a wide range of electronic circuits. They had their origins in analog computers where they were used in many linear, non- linear and frequency-dependent circuits. Op-amps are among the most widely used electronic devices today, being used in a vast array of consumer, industrial, and scientific devices. The modern operational amplifier is a solid state, high gain, DC voltage amplifier. In this paper we design a virtual SoftLab to observer the following application using VIS Model.

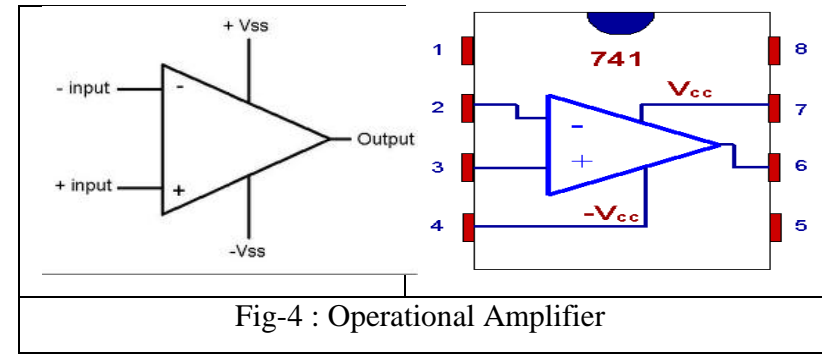

The Inverting Amplifier: In an inverting amplifier, the output voltage changes in an opposite direction to the input voltage.

The Non-Inverting Amplifier: In a non-inverting amplifier, the output voltage changes in the same direction as the input voltage

Integrator: An integrator is a circuit that performs a mathematical operation called integration. The most popular application of an integrator is in producing a ramp of output voltage, which is a linearly increasing or decreasing voltage.

Differentiator: A differential combines the two ac collector voltages into one voltage that equals the difference of the collector voltages.

Inverting Adder: Summing amplifier combines two or more analog signals into a single output. For simplicity, the circuit shows only two inputs, but we can have as many inputs as needed for the application

A program is constructed for conduct of Operational Amplifier experiment in VIS in 'VB' such that all the blocks in the model can be fully visualized on the screen. This model also can demonstrate the activities of Operational Amplifier including circuit connection visually. Inputs accepted through virtual signal generator which is observable on screen. In an experiment, we study of operational amplifier and observed virtual outputs. This model provides circuit connection facility to user so that the user can practice circuit connection also.

\subsection{DESIGN SPECIFICATIONS}

An Operational Amplifier VIS Model requires virtual sine wave generators, virtual $\mathrm{CRO}$ and virtual connection wires. Virtual sine wave generator allowed different setting of amplitude and frequency wave signal. The virtual instruments required for complete experimental setup like virtual AC supply, carrier wave generator, modulating wave generator, CRO and DC power supply are visible on the screen. With this arrangement we can perform all possible operations such as Inverting, Non-Inverting, Integrator, Differentiator, Voltage Followers and Inverting Adder.

Important screen shots of the experiment which appear on the monitor screen are shown in Fig-5, Fig-6, Fig-7, and Fig-8. 


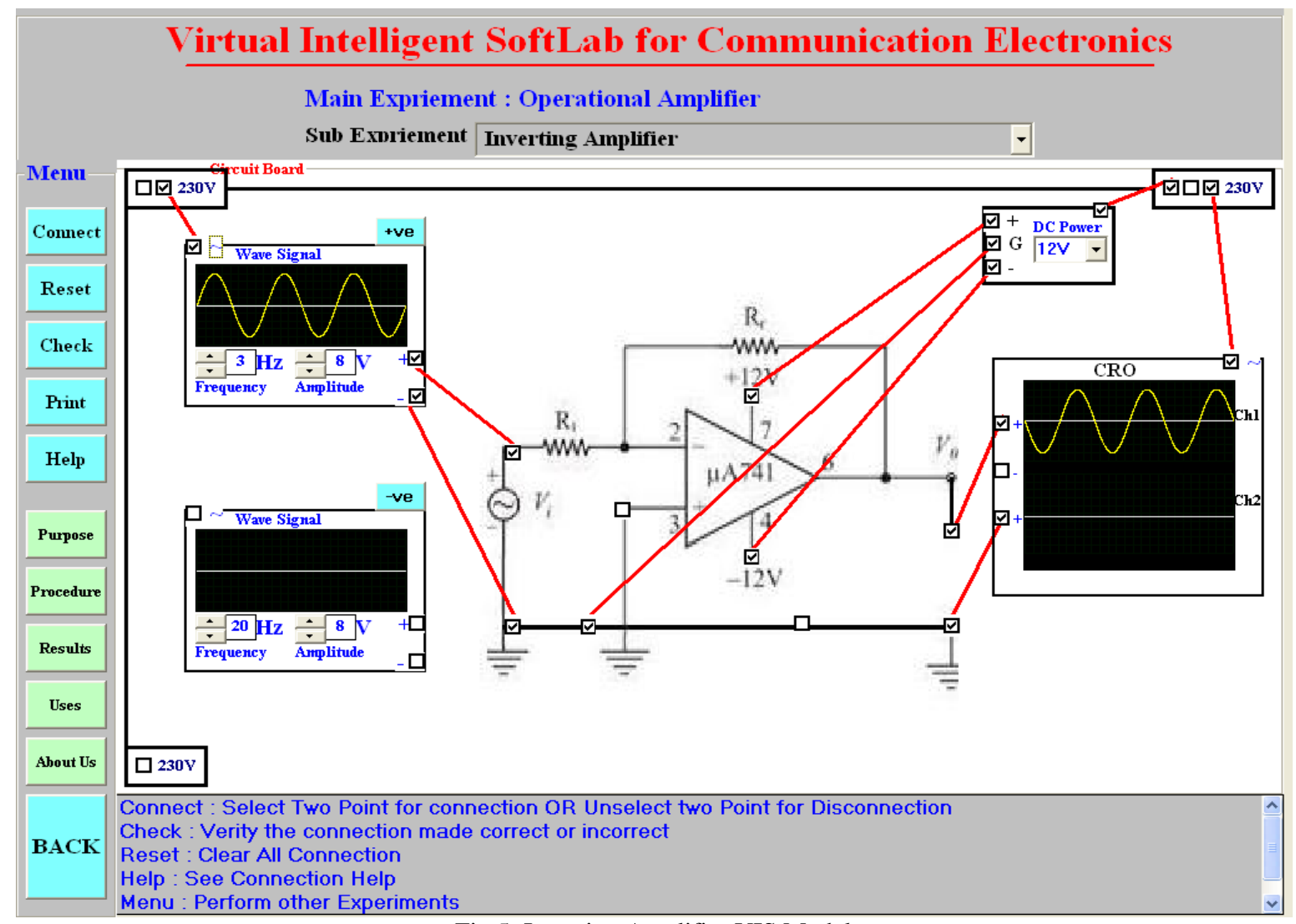

Fig 5: Inverting Amplifier VIS Model

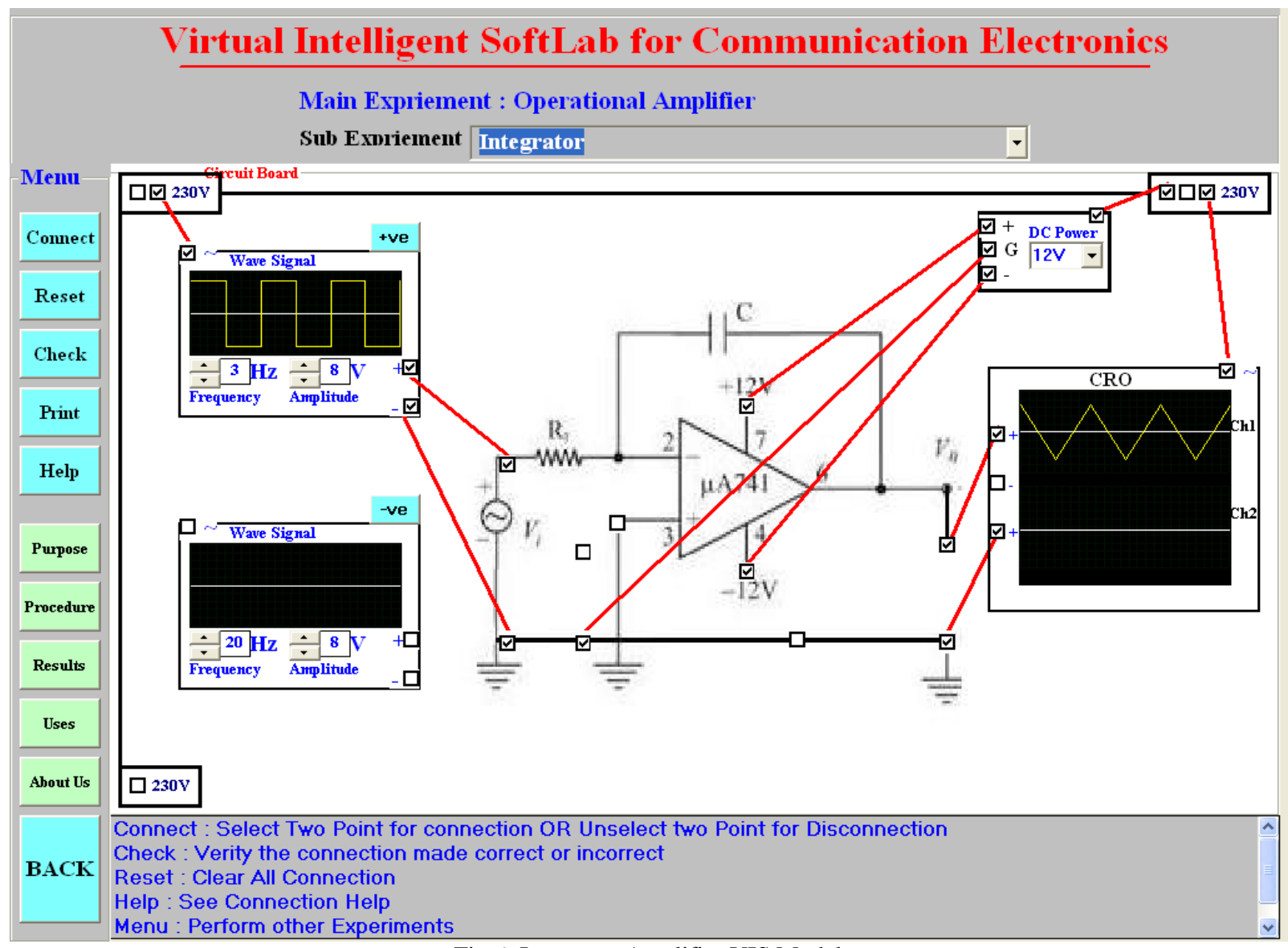

Fig 6: Integrator Amplifier VIS Model 


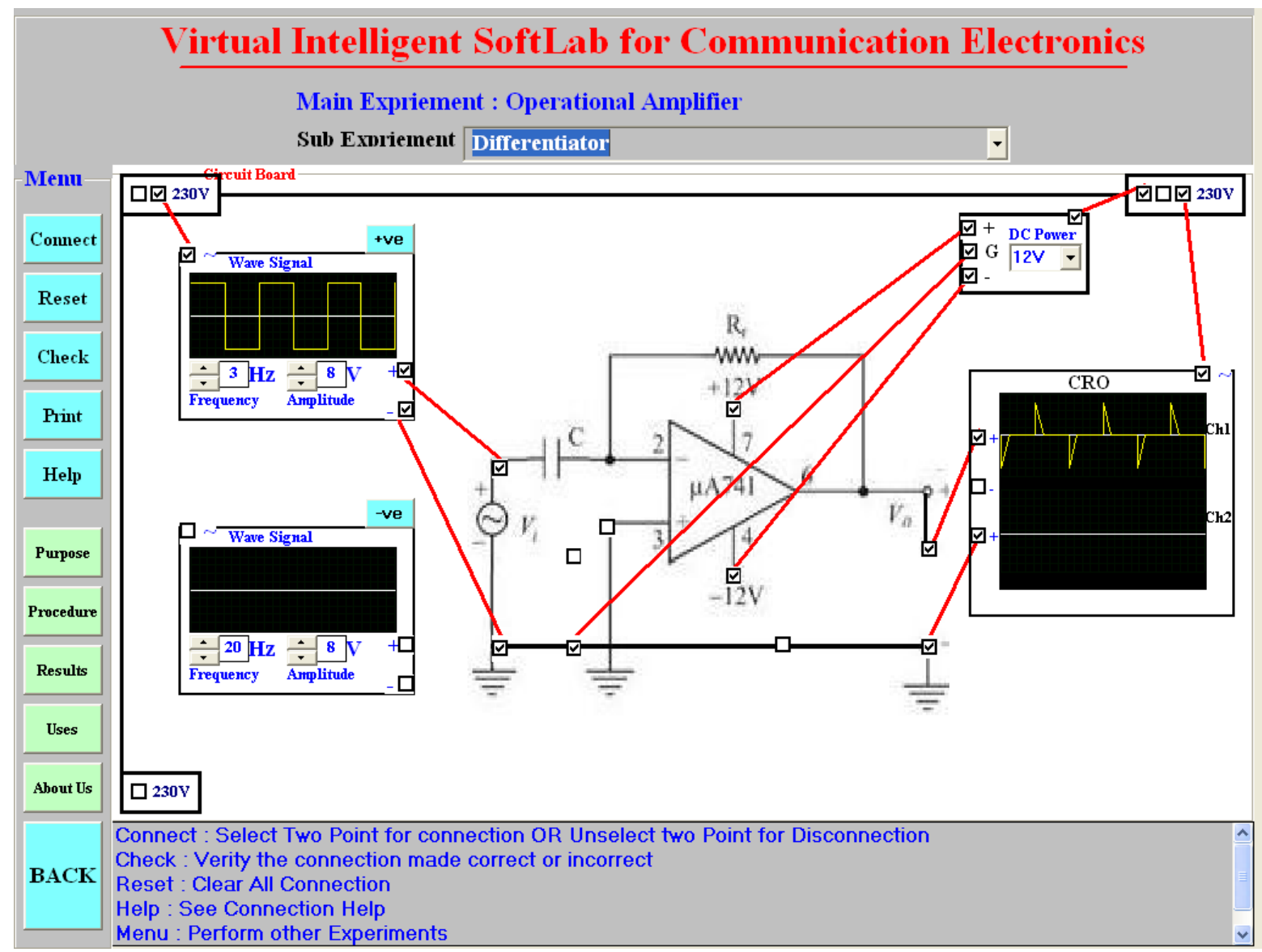

Fig 7: Differentiator Amplifier VIS Model

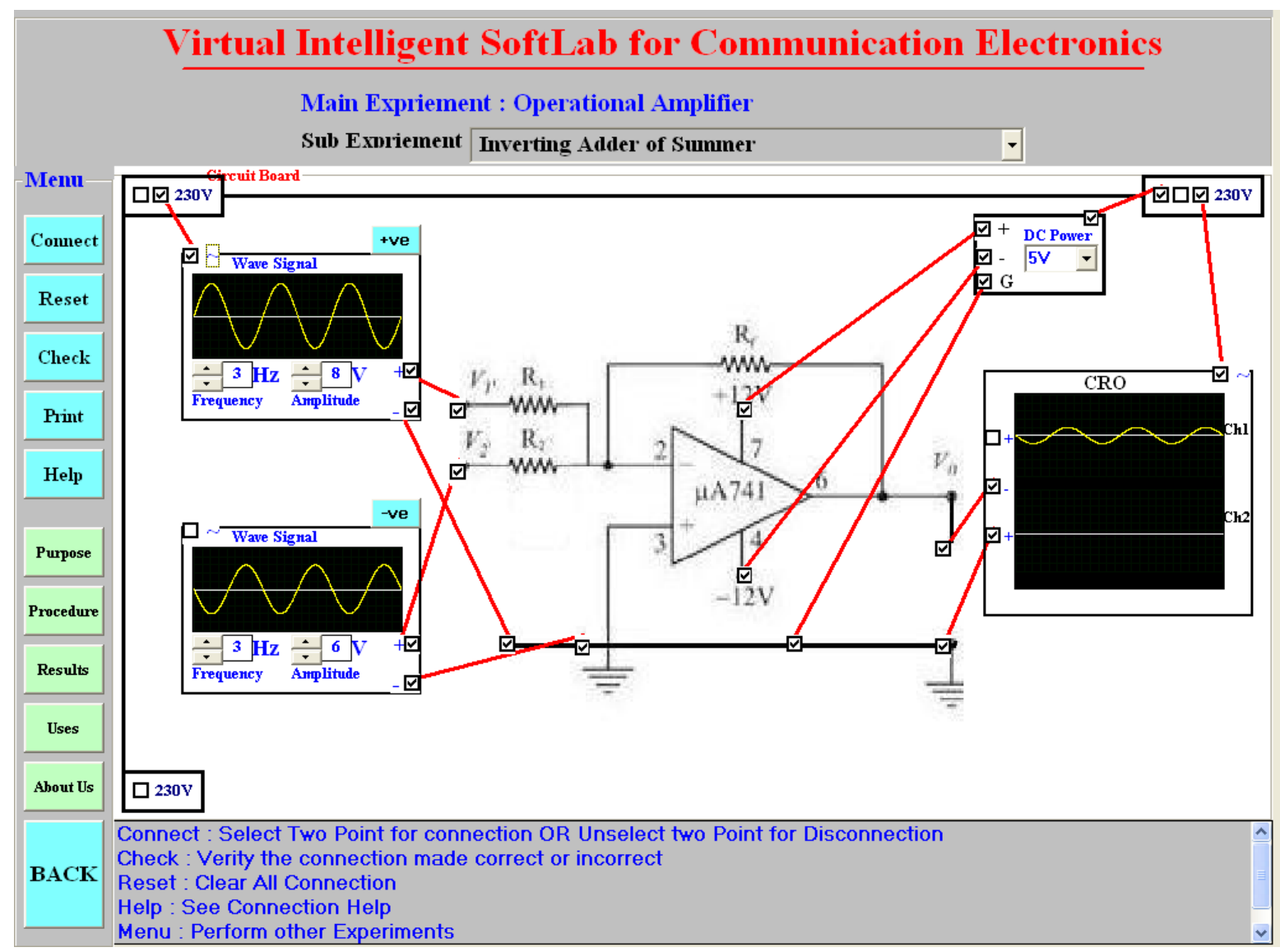

Fig 8: Inverting Adder Amplifier VIS Model 


\subsection{Implementation}

To perform the experiment with VIS Model one has to go through the following steps.

Step: 1. Connect AC socket to DC Converter Device.

Step: 2. Connect DC power supply to $\mathrm{V}_{\mathrm{cc}}$ pin.

Step: 3. Connect Ground Socket to Ground Pin

Step: 4. Connect AC power supply to virtual sine Wave generators and CRO.

Step: 5. Change Amplitude and/or frequency and observe output on CRO.

This model is user friendly. Here, the instructor/student can access the detail procedure to connect the circuit. This procedure includes following steps -

- Select two switches for connection.

- Click the "Check Button" to verify the connection.

- Click the "Reset Button" if the connections are WRONG.

- Click the "Help Button" if you need Connection HELP

- Click the "Menu Button" if you want to perform other Experiments

\subsection{Results}

Effect of change in input signal and output signal is observable using operational amplifier. The VIS Management System was implemented in the laboratory of the Institution of Electronics and Telecommunication Engineers, Nagpur Centre. In all thirty students performed all the experiments designed in VIS Model.

\section{CONCLUSIONS}

SoftLab will help Electronics, Computer Science and Engineering students to perform and practice experiments to improve their understanding of the subject. The design of the VIS model is more effective and realistic as necessary variable inputs and outputs are visible on the monitor screen. Virtual experiments easily perform with Virtual SoftLab without physical damages and observed the real outputs. Virtual instruments reduce the instrument cost and laboratory setups. This virtual experiment provides practice to students for the 'touch \& feel' part they have already performed in the laboratory.

\section{ACKNOWLEDGEMENT}

We are very much thankful to Dr. U. A. Lanjewar, Professor, P. C. D. I.C.S.R. V.M.V. College campus for his valuable inputs, constant guidance and his extensive support an encouragement for this work.

\section{REFERENCES}

[1]. "Virtual Labs:" Nov 2010, http://www.vlab.co.in/

[2]. “A Virtual Laboratory” March 2011; http://www.virtlab .com/main.aspx

[3]. N. D. Deshpande, D. A. Deshpande and P.K. Rangole, Communication Electronics, Tata McGraw-Hill, 2000.

[4] Aynur Akar and Ayse Yayla (2011). Web Based Real Time Laboratory Applications of Analog and Digital Communication Courses with LabVIEW Access, Modeling, Programming and Simulations Using LabVIEW ${ }^{\mathrm{TM}}$ Software, Dr Riccardo De Asmundis (Ed.), ISBN: 978-953-307-521-1, InTech, Available from: http://www.intechopen.com/books/modelingprogramming-and-simulations-using-labviewsoftware/web-basedreal-time-laboratory-applications-ofanalog-and-digital-communication-courses-with-labview

[5] SoftLab- A Virtual Laboratory for Computational Science 1 (1980), By CM Hoffman

[6]. Handbook of Operational Amplifier Application by Bruce Carter and Thomas R. Brown, Application Report, SBOA092A -October 2001

[7] Malvino, Electronic Principle, McGraw-Hill, 1999

[8] Paul Horowitz and Winfield Hill, "The Art of Electronics 2nd Ed. " Cambridge University Press, Cambridge, 1989 ISBN 0-521-37095-7

[9] Sergio Franco, "Design with Operational Amplifiers and Analog Integrated Circuits," 3rd Ed. McGraw-Hill, New York, 2002 ISBN 0-07-232084-2 